\title{
Bi-layer metamaterials as fully functional near-perfect infrared absorbers
}

\author{
Bryan M. Adomanis, ${ }^{1, a)}$ Claire M. Watts, ${ }^{2}$ Machhindra Koirala, ${ }^{2, b)}$ Xianliang Liu, ${ }^{2}$ \\ Talmage Tyler, ${ }^{3}$ Kevin G. West, ${ }^{4}$ Tatiana Starr, ${ }^{4}$ Jonathan N. Bringuier, ${ }^{4}$ Anthony F. Starr, ${ }^{4}$ \\ Nan Marie Jokerst, ${ }^{3}$ and Willie J. Padilla ${ }^{2, c)}$ \\ ${ }^{1}$ Air Force Research Laboratory, Wright-Patterson AFB, Ohio 45433, USA \\ ${ }^{2}$ Department of Physics, Boston College, Chestnut Hill, Massachusetts 02467, USA \\ ${ }^{3}$ Department of Electrical and Computer Engineering, Duke University, Durham, North Carolina 27708, USA \\ ${ }^{4}$ SensorMetrix, Inc., San Diego, California 92121, USA
}

(Received 20 March 2015; accepted 26 June 2015; published online 16 July 2015)

\begin{abstract}
In this letter, we discuss the design, fabrication, and experimental characterization of a bi-layer fully functional near-perfect metamaterial absorber (MMA) in the long-wavelength infrared (LWIR), which is broadband and generally insensitive to polarization up to a $60^{\circ}$ incidence angle. A spectral absorptance of $\geq 99 \%$ was attained simultaneously at multiple LWIR wavelengths, with a bandwidth of $2 \mu \mathrm{m}$ where the absorptance is $\geq 90 \%$. This remarkable behavior is attributed to the strong mixing of coupling modes between the two resonators and the ground plane in the presence of a lossy dielectric, in which single layer structures do not exhibit. Furthermore, we show, by comparing two different MMA structures, how the absorption can be tailored by design within and across several IR subdivisions through a slight change in geometrical parameters. The bi-layer MMA has the immediate application of a functionally versatile, low-profile thermal sensor or emitter. (C) 2015 AIP Publishing LLC. [http://dx.doi.org/10.1063/1.4926416]
\end{abstract}

Perfect absorbers in the infrared (IR) are particularly exciting due to their applicability as thermal emission control surfaces, among other applications. Efforts have been made to tailor the thermal emission of structures through the use of 1-D and 2-D gratings, ${ }^{1,2}$ photonic crystals, ${ }^{3,4}$ and microstrip patch arrays. ${ }^{5}$ However, many of these solutions are narrowband and lack functionality at certain polarizations or incidence angles, all of which are critical to practical thermal applications where maximum absorption of light is desired. ${ }^{6}$

Since the experimental demonstration of a near-unity metamaterial absorber (MMA) in the microwave spectrum in $2008,{ }^{7}$ a great deal of research has been invested into establishing the utility of a perfect absorber for real-world application. Work rapidly expanded into the terahertz, ${ }^{8}$ short-wave IR, ${ }^{9}$ mid-wave IR, ${ }^{10}$ near-infrared, ${ }^{11,12}$ and visible range, ${ }^{13}$ and with it came a flood of attempts to increase bandwidth and insensitivities to polarization and incidence angles (see Ref. 6 for a comprehensive review). Initially, most of these designs suffered in one area or another: multi-band and wideangle designs were still narrow-band ${ }^{14-17}$ or polarization-dependent, ${ }^{7,8}$ while these wholly insensitive broadband designs did not attain a consistent absorptance over 90\%.18,19 However, significant advancements have been made in creating "fully functional" near-perfect absorbers: those which exhibit strong broadband responses while being simultaneously angle and polarization insensitive. In the past year alone, fully functional MMAs have been demonstrated in the microwave, ${ }^{20-25}$ terahertz, ${ }^{26-28}$ and optical ${ }^{29}$ regimes. Despite this

\footnotetext{
${ }^{\text {a)} E l e c t r o n i c ~ m a i l: ~ b r y a n . a d o m a n i s @ u s . a f . m i l . ~}$

b) Present address: Department of Physics and TcSUH, University of Houston, Houston, Texas 77204, USA.

${ }^{c)}$ Present address: Department of Electrical and Computer Engineering, Duke University, Durham, North Carolina 27708, USA. Electronic mail: willie.padilla@duke.edu.
}

advancement, we found a lack in development of fully functional IR MMAs. ${ }^{30-38}$

In this letter, we demonstrate, both computationally and experimentally, a fully functional near-perfect MMA in the long wavelength IR (LWIR) range. We used a bi-layer pattern to achieve a wide-angle, polarization-insensitive, broadband performance which is superior overall to many single layer $^{30,31,33,35}$ and previous multi-layer nested designs ${ }^{39}$ in the IR regimes and which is comparable to state-of-the-art in other frequency regimes cited above. Experimentally, we achieve an absorptance of up to $99.9 \%$ with a $90 \%$ absorptance bandwidth of over $2 \mu \mathrm{m}$ or about $20 \%$ of the center LWIR wavelength of $10 \mu \mathrm{m}$. For both sand ppolarizations, the broadband absorption remains approximately at or above $90 \%$ up to $45^{\circ}$ angles and at or above $80 \%$ up to $60^{\circ}$ angles.

Invoking Kirchhoff's law and ignoring cross-polarization terms and diffraction beyond zeroth-order (verified to be negligible in our parameter space), the spectral directional absorptance is described by $A_{s / p}(\omega, \theta)=11-T_{s / p}(\omega, \theta)$ $-R_{s / p}(\omega, \theta)$, where $\omega$ is the angular frequency, $\theta$ is the polar angle of incidence, $T$ and $R$ are the squares of the complexvalued Fresnel transmission and reflection coefficients, respectively, and $s / p$ identifies the $\mathrm{s}$ - or p-polarization state and accounts for the azimuthal $(\phi)$ dependence on the absorption. The total hemispherical absorptance can then be found by integrating over the spectrum and hemispherical space and then summed for each polarization. Therefore, this bi-layer MMA, as a fully functioning absorber of LWIR radiation, should act as a more intense thermal emitter over absorbers which are limited in bandwidth, polarization, or angle, as long as absorption can remain strong throughout the parameter space.

Consequently, one can create strong absorption by simultaneously diminishing the reflection and transmission 
responses of a structure. The conventional design of a planar MMA consists of a single electric resonator on a ground plane separated by a lossy dielectric layer, which can ensure $T \rightarrow 0$ and minimize back reflections. For minimization of reflection off the top surface, impedance matching to free space is required, i.e., such that $Z(\omega, \theta)$ $=\sqrt{(\mu}(\omega, \theta) / \varepsilon(\omega, \theta)=Z_{0}=120 \pi \Omega .{ }^{40}$ Here, effective medium theory for metamaterials can be employed, where the geometry of the MMA structure determines the complex optical constants: the electric permittivity $\varepsilon(\omega)$ and the magnetic permeability $\mu(\omega) .{ }^{41}$ The electric response is achieved through the metallic resonator and the magnetic response is achieved through antiparallel currents in the top layer and the ground plane. Tuning of the electric and magnetic responses can be achieved by changing the geometry of the resonator and varying the thickness of the dielectric, respectively, giving near unity absorptance at the impedance matched wavelength. ${ }^{6}$

However, this approach is not suitable for the preponderance of multi-band designs which are single layer, i.e., the electric resonators all lie in the same plane, in a "wallpaper" fashion. ${ }^{19,23,30,42-45}$ One drawback is that the filling fraction of electric resonators is reduced over the single band case. Another drawback is that the magnetic response must be tuned for all electric resonators simultaneously, rather than individual tuning. In single-plane designs, one $\mu$ determined by the dielectric thickness contributes to $Z(\omega, \theta)$ for all resonators, allowing for the perfect impedance match of at best one of the resonators to free space. The result is an imperfect impedance match, and hence imperfect absorption, for each frequency band. The bi-layer design presented in this letter provides a significant advantage over traditional single layer broadband devices because not only is the filling fraction maximized, but also because impedance matching is only required for the upper interface resonator, allowing individual tuning of not only the electric response via the geometry of the stacked metallic resonators but now also the magnetic response through the thickness of each dielectric layer.

Using the finite-element computational software COMSOL Multiphysics, ${ }^{46}$ we have designed two different fully functional MMA structures for demonstration. Topview and side-view schematics and scanning electron microscope (SEM) images of the MMA structure are shown in Figures 1(a)-1(d). Each structure comprised three metallic elements-two stacked gold cross resonators over a gold ground plane-each separated by benzocyclobutene (BCB) dielectric spacers. Several parameters are identical for both structures: a unit cell size of $a=3.2 \mu \mathrm{m}$, a ground plane of thickness of $100 \mathrm{~nm}$, and each resonator consists of a $50 \mathrm{~nm}$ thick gold pattern with line width $w=500 \mathrm{~nm}$. The sizes of the resonators in structure 1 (S1) are $l_{B}=2.8 \mu \mathrm{m}$ for the lower resonator and $l_{T}=2.7 \mu \mathrm{m}$ for the top resonator, which generate slightly different resonance frequencies, creating a broadband effect. The bottom resonator is separated from the ground plane by a BCB layer $t_{1}=170 \mathrm{~nm}$ and the two resonators are separated by $t_{2}=500 \mathrm{~nm}$. Structure 2 (S2) exhibits a slight reduction in these parameters, with $l_{B}=2.7 \mu \mathrm{m}$ and $l_{T}=2.65 \mu \mathrm{m}$, and with the BCB layers having thicknesses $t_{1}=160 \mathrm{~nm}$ and $t_{2}=350 \mathrm{~nm}$. Neither design is

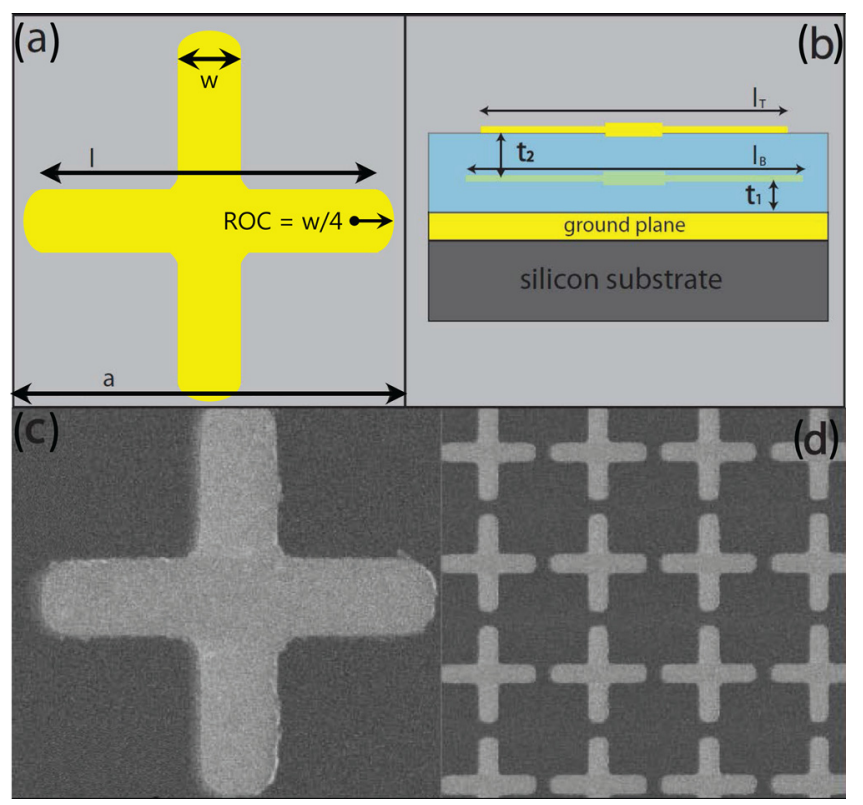

FIG. 1. Design and fabrication of bi-layer MMA. (a) Schematic diagram of top view of unit cell. (b) Side view of the MMA unit cell. Blue represents the BCB dielectric spacer, grey represents the silicon substrate, and yellow represents the gold metamaterial and ground plane layer. (c) SEM image of the unit cell. (d) The SEM image of the periodic array.

optimized beyond the interest of a near-perfect absorption around $10 \mu \mathrm{m}$ - though it was assumed the gold cross dipole would perform favorably over a wide set of parameters, no materials or structural optimization steps were applied in the design to maximize the response.

The frequency dependent complex refractive index for gold is based on Ordal, ${ }^{47}$ while the $\mathrm{BCB}$ properties are extracted using ellipsometric techniques. The spectral behaviors for both polarization states are calculated as $T(\omega, \theta)$ $=\left|S_{21}(\omega, \theta)\right|^{2}$ and $R(\omega, \theta)=\left|S_{11}(\omega, \theta)\right|^{2}$, where $S_{21}$ and $S_{11}$ denote the complex Fresnel transmission and reflection coefficients, respectively, for a reciprocal scattering network. Floquet boundary conditions are applied to a single unit cell to simulate an infinite periodicity and to account for phase shift across the elements at oblique incidence angles. The absorptance $A(\omega, \theta)$ is calculated by $1-R(\omega, \theta)$, due to the presence of the electrically thick and continuous metallic ground plane. In the stated periodicity, only the -1 st-order diffraction mode exists throughout the angular range, up to $6.29 \mu \mathrm{m}$ at $75^{\circ}$. By defining this as the lower wavelength limit of what is presented in simulation, diffraction can be ignored in the calculation for $A(\omega, \theta)$.

Fabrication started with two 6-in. $\langle 100\rangle$ silicon wafers to serve as substrates. First, the gold ground plane is deposited via electron-beam evaporation. The first $\mathrm{BCB}$ dielectric layer is then spin-coated and cured; the wafers are prepared with varying $B C B$ thicknesses of $160 \mathrm{~nm}$ and $170 \mathrm{~nm}$, as stated above. The first meta-material layer is then photolithographically patterned using a deep UV $(193 \mathrm{~nm})$ step and scan system (at the North Carolina State University Nanofabrication Facility, NNF). A $50 \mathrm{~nm}$ gold film is then evaporated and the metamaterial patterns defined using the lift-off technique. The second dielectric BCB layer is spincoated and cured; the second $\mathrm{BCB}$ layer serves as the dielectric as well as a planarizing layer, which is necessary for 
subsequent photo-lithographic patterning. The second metamaterial layer is formed in a manner similar to the first. The samples were shown to have excellent uniformity and alignment [see Figures 1(c) and 1(d)].

Spectral reflectance measurements were carried out using the SOC-100 Hemispherical Directional Reflectometer (HDR), developed by Surface Optics Corporation. ${ }^{48}$ A Lambertian thermal source is positioned at one focus of a gold-plated hemi-ellipsoidal cavity, and the sample is placed at the other focus, thereby illuminating the sample from all angles. The collection optics capture the polarized diffuse and specular components of the scattered energy, providing directional reflectances from incidence polar angles between $8^{\circ}$ and $80^{\circ}$. All reference to normal incidence measurements were taken at this minimum angle. Transmission and diffuse reflection were validated by cursory measurements to be negligible contributions within the desired spectrum and angular range, which validates our numerical approach.

Both the experimental (blue, solid) and simulated (red, dot) absorptances at normal incidence for S1 and S2 are shown in Figures 2 and 3, respectively. In both structures, we see several primary peaks between the approximate wavelength range of 8.5-10.5 $\mu \mathrm{m}$. These resonances overlap enough to form a band of near unity absorptance, with the structures exhibiting a bandwidth of absorptance over $90 \%$ of $2.08 \mu \mathrm{m}$ and $1.84 \mu \mathrm{m}$, respectively. In S1, there are three wavelengths of near unity absorptance at $9.14 \mu \mathrm{m}, 9.93 \mu \mathrm{m}$, and around $10.5 \mu \mathrm{m}$, with experimental absorptances of $99.9 \%, 99.8 \%$, and $\sim 97 \%$, respectively, which generate an impressive $95 \%$ bandwidth of $1.81 \mu \mathrm{m}$. Due to the altered geometry, S2 has slightly blue-shifted absorptance peaks at $8.57 \mu \mathrm{m}, 8.93 \mu \mathrm{m}$, and $9.99 \mu \mathrm{m}$ with experimental absorptances of $99.9 \%, 99.0 \%$, and $99.0 \%$, respectively. Note that at the designed absorption wavelength of $10 \mu \mathrm{m}$, there are nearly equal contributions of resistive and Ohmic losses.

The simulated responses (at true normal incidence) are in good agreement with experimental data, with the largest discrepancies being reductions in some regions of the simulated peak amplitudes on the order of about $10 \%$. We assert

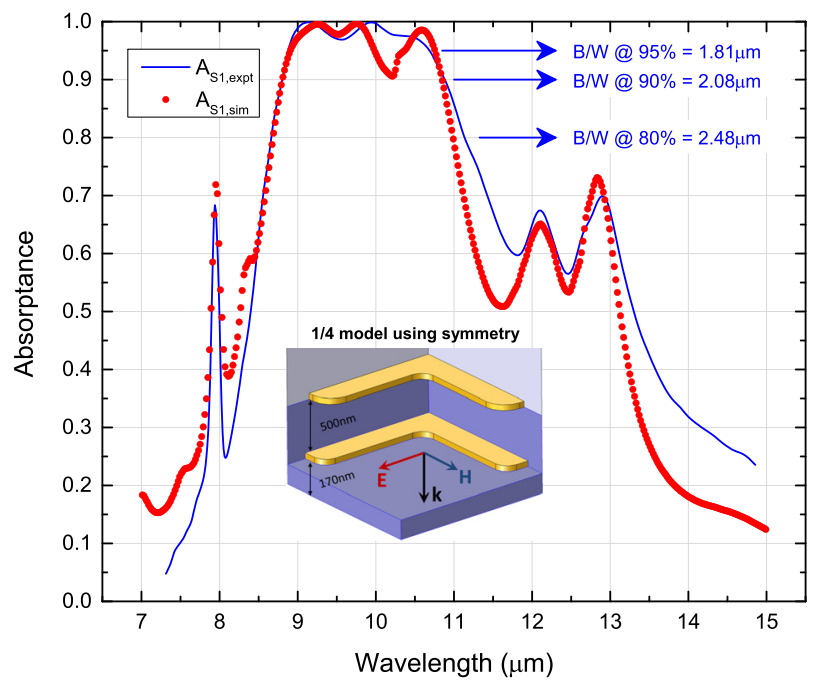

FIG. 2. Experimental (solid) and simulated (dot) spectral absorptance at normal incidence for S1. Inset image shows modeled structure, taking advantage of four-fold symmetry.

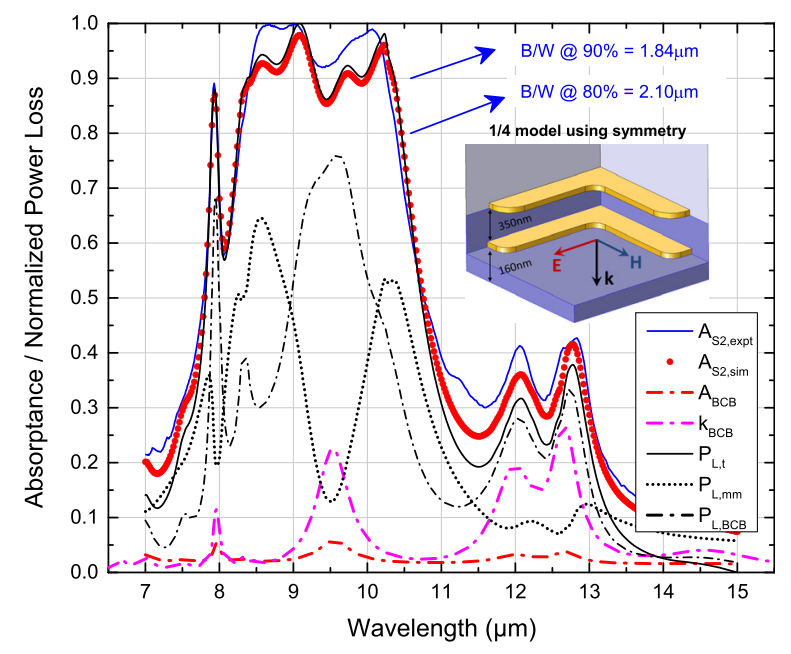

FIG. 3. Same results as in Figure 2, for S2. The power loss is extracted from separate volume integrals of the power loss density in the gold metamaterial (black, dot) and BCB (black, dash-dot) domains and are normalized to the total power loss (black, solid) in both domains. Extracted data of the BCB imaginary component of the refractive index, $k$ (magenta) and the simulated absorptance of unadorned $\mathrm{BCB}$ (red, dash-dot) show how power loss is magnified in the dielectric by presence of the metamaterial inclusion.

this variance to be primarily due to the inaccuracy of the amplitudes and peak positions of the Lorentzian oscillators fitted from the BCB parameter extraction technique, and highlight the sensitivity of these properties on the model. This assertion can be qualifiedly justified by examining the imaginary component of the refractive index, $k$, of the $\mathrm{BCB}$ substrate, displayed in Figure 3 (magenta, dash-dot), and comparing against recent work on BCB thin films by Kamineni et al. ${ }^{49}$ While the overall agreement between our $\mathrm{BCB}$ properties are good, there is a significantly reduced (but present) peak in our data near $10 \mu \mathrm{m}$ that would likely account for the discrepancy in this spectral region if it were set at this higher value.

As there are two metal resonators, it is expected that the absorptance could be attributed to the mixed coupling states between the two resonators, the upper or lower resonator and the ground plane, and both resonators with the ground plane in various degrees. In addition, another source of high spectral absorption would be accomplished from the energy exchange between these elements in the lossy dielectric. To better understand the source of this broad absorptance, we explored the power dissipation in the simulated S2 MMA. Figure 3 shows the power loss in the gold metamaterial, $P_{L, m m}$ (black, dot) and in the BCB, $P_{\mathrm{L}, \mathrm{BCB}}$ (black, dash-dot), derived from volume integrals of the power dissipation density in the metal and dielectric domains, respectively. These values were normalized to the total $P_{L, t}$ (black, solid). As expected, both resistive and dielectric losses exist, with the former appearing as minima at the maxima of the latter, but not necessarily vice versa. Peaks of $P_{L, m m}$ appear where the maximum absorptances exist for a structure with a single, isolated metamaterial of identical geometry and position (not shown), with the peak at $8.55 \mu \mathrm{m}$ representing the upper inclusion and the peak at $10.24 \mu \mathrm{m}$ representing the lower one. Peaks of $P_{L, B C B}$ follow $k$, as well as the simulated absorptance in a undecorated slab (i.e., no metamaterial) of the BCB substrate (red, dash-dot), which, when compared to the 
MMA absorptance, serves as a useful demonstration of the effective magnitude of introducing the resonant metamaterial into the system.

The particular coupling behavior between the resistive elements can be identified by exploring the power dissipation and current densities in the structure at these peaks, shown in Figure 4. Black cones represent the displacement current density $\left(\mathrm{A} / \mathrm{m}^{2}\right)$, and the surface colormap denotes the power dissipation density $\left(\mathrm{W} / \mathrm{m}^{3}\right)$. At the narrow peak of $P_{L, B C B}(\lambda=7.93 \mu \mathrm{m})$ (a), energy from the upper resonator scatters into the $\mathrm{BCB}$, but is dissipated largely in the substrate without effectively coupling to the lower resonator or ground plane. Conversely, at $P_{L, m m}(\lambda=8.55 \mu \mathrm{m})$ (b), the low material loss permits strong coupling to the lower resonator, generating a circular current flow, which in turn couples more weakly to the ground plane. This generates a field concentration in the BCB near the metamaterial surface, which contributes significantly to a large $A$, even in a region of low material loss. At the peak of $P_{L, B C B}(\lambda=9.60 \mu \mathrm{m})$ (c), the losses in the metamaterial have visibly diminished and there are only weak couplings between the resistive elements; yet, the dissipation remains large, as what energy is accepted into the MMA couples primarily into the BCB phonon mode. At the other resistive peak at $P_{L, m m}(\lambda=10.24 \mu \mathrm{m})(\mathrm{d})$, there is a strong coupling between the lower resonator and the ground plane, with little interaction with the upper resonator. Overall, these results show an absorption not only via the conventional impedance matching condition but also through the mutual interaction of all three layers. This understanding of the coupling mechanisms and the interplay between resistive and dielectric losses can be utilized in optimizing the structure further such as improving bandwidth.

Figures 5(a) and 5(b) show the experimental and simulated angular absorptance spectra of $\mathrm{S} 2$ at $15^{\circ}$ intervals, from a p-polarized and s-polarized plane wave, respectively. For both polarizations, the experimental absorptance remains above $90 \%$ for a majority of the region between 8.25 and $10.0 \mu \mathrm{m}$, up to $45^{\circ}$. The p-polarized absorptance remains broad at around $80 \%$ up to $60^{\circ}$, whereas the s-polarized

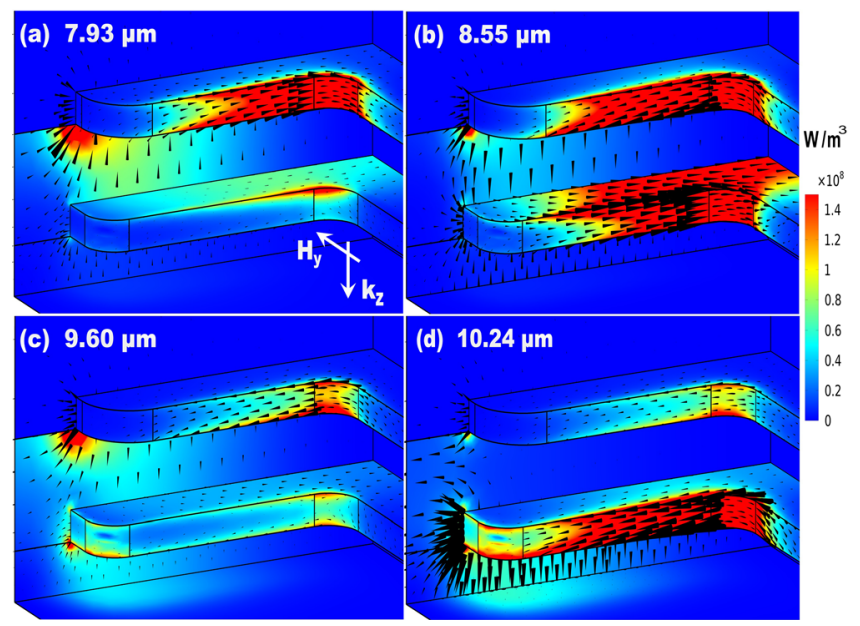

FIG. 4. Simulated distributions of power loss density (colormap), and displacement current density, $J_{d}$ (black cones), for a quadrant of S2, sampled at the strong dielectric ((a) and (c)) and resistive ((b) and (d)) peaks shown in Figure 3 . In the dielectric domains, $J_{d}$ magnitudes are amplified by a factor of 20 from that at the metal interface.

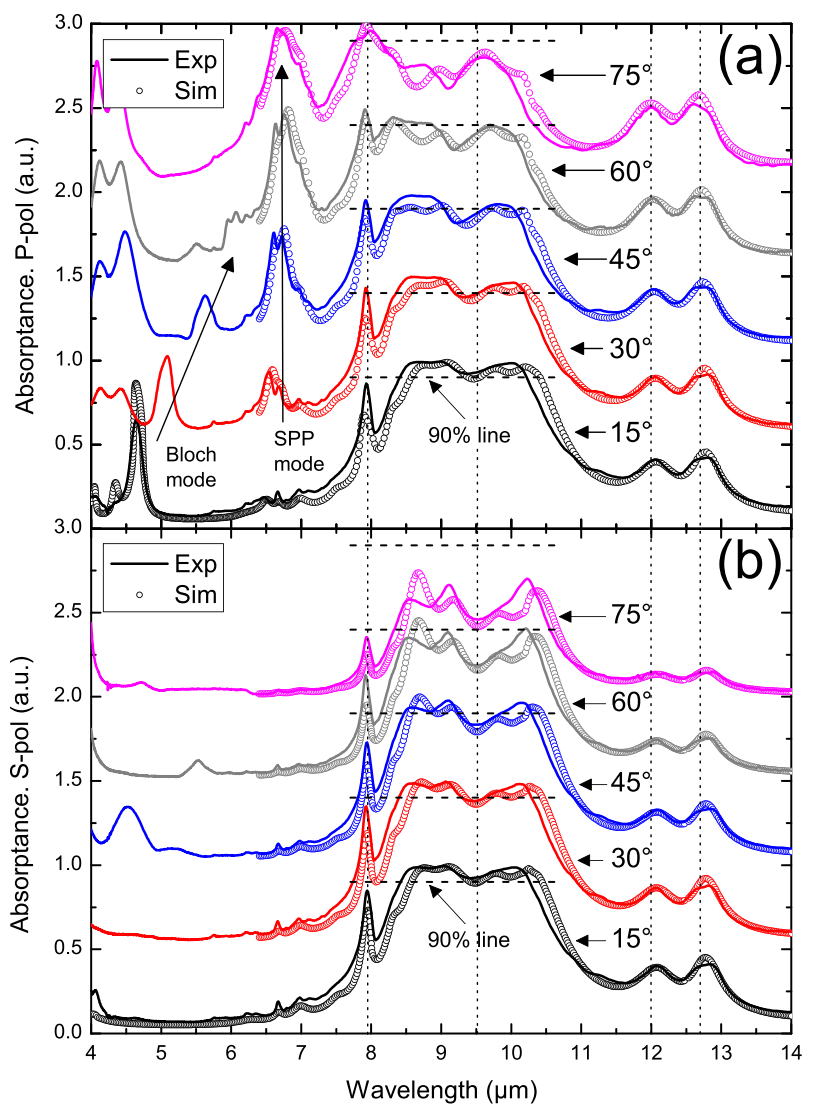

FIG. 5. Experimental (solid) and simulated (dotted) spectral absorptance of $\mathrm{S} 2$ at oblique angles, in $15^{\circ}$ intervals, from (a) p-polarized and (b) s-polarized incident light. Amplitudes are offset by 0.5 for each angle, so the dashed line represents $90 \%$ absorptance for each data set. Vertical lines represent the $\mathrm{BCB}$ absorption peaks.

response begins to drop, but is generally $\geq 50 \%$ up to $60^{\circ}$. This is because the p-polarized light maintains a strong magnetic coupling at high angles, while magnetic coupling of the s-polarized light becomes progressively weaker with increasing angle.

Two other peaks can be seen in Figure 5(a) that do not appear in Figure 5(b). One is a surface plasmon polariton (SPP) mode which reaches near-perfect absorptance around $6.6 \mu \mathrm{m}$, and the other is a Bloch mode observable around $4.75 \mu \mathrm{m}$ which predictably red-shifts with increasing angle and ultimately merges into the SPP mode (not simulated, as mentioned above, except at $15^{\circ}$-where there is only a 0 th order response- to show agreement). Both resonances have been reported in our previous works on single layer MMAs. ${ }^{50}$

In conclusion, we have presented the design, fabrication, and characterization of a fully functional MMA in the LWIR regime based on a bi-layer structure. We achieved at normal incidence a broadband absorptance with a bandwidth of $90 \%$ spanning over $2 \mu \mathrm{m}$ ( $20 \%$ bandwidth at LWIR center band) and a maximum experimental spectral absorptance of $99.9 \%$ that is generally insensitive up to $45^{\circ}$. The enhanced absorption is due to the variable interlayer coupling mechanisms within the lossy dielectric, the increase in filling fraction and the ability to accurately tune the electric and magnetic resonances for each layer. This low-profile MMA structure could easily be used for a broadband, wide field-of-view sensor or emitter over a span of desired IR subdivisions. 
We would like to acknowledge partial funding from Air Force Office of Scientific Research under U.S. Air Force Contract No. FA9453-13-C-0005 and U.S. Navy Contract No. N00014-10-C-0437, and we would like to thank the Air Force Institute of Technology for support on HDR measurements.

${ }^{1}$ J.-J. Greffet, R. Carminati, K. Joulain, J.-P. Mulet, S. Mainguy, and Y. Chen, Nature 416, 61 (2002).

${ }^{2}$ C. Arnold, F. Marquier, M. Garin, F. Pardo, S. Collin, N. Bardou, J.-L. Pelouard, and J.-J. Greffet, Phys. Rev. B 86, 035316 (2012).

${ }^{3}$ F. G.-V. E. Moreno and L. Martín-Moreno, Phys. Rev. B 69, 121402 (2004).

${ }^{4}$ P. Kramper, M. Agio, C. M. Soukoulis, A. Birner, F. Müller, R. B. Wehrspohn, U. Gösele, and V. Sandoghdar, Phys. Rev. Lett. 92, 113903 (2004).

${ }^{5}$ I. Puscasu and W. L. Schaich, Appl. Phys. Lett. 92, 233102 (2008).

${ }^{6}$ C. M. Watts, X. Liu, and W. J. Padilla, "Metamaterial electromagnetic wave absorbers," Adv. Mater. 24(23), OP98-120 (2012).

${ }^{7}$ N. I. Landy, S. Sajuyigbe, J. J. Mock, D. R. Smith, and W. J. Padilla, Phys. Rev. Lett. 100, 207402 (2008).

${ }^{8}$ H. Tao, C. M. Bingham, A. C. Strikwerda, D. Pilon, D. Shreken-hamer, N. I. Landy, K. Fan, X. Zhang, W. J. Padilla, and R. D. Averitt, Phys. Rev. B 78, 241103 (2008).

${ }^{9}$ Y. Avitzour, Y. A. Urzhumov, and G. Shvets, Phys. Rev. B 79, 045131 (2009).

${ }^{10}$ X. Liu, T. Starr, A. F. Starr, and W. J. Padilla, Phys. Rev. Lett. 104, 207403 (2010).

${ }^{11}$ J. Hao, J. Wang, X. Liu, W. J. Padilla, L. Zhou, and M. Qiu, Appl. Phys. Lett. 96, 251104 (2010).

${ }^{12}$ K. B. Alici, A. B. Turhan, C. M. Soukoulis, and E. Ozbay, Opt. Express 19, 14260 (2011).

${ }^{13}$ C.-H. Lin, R.-L. Chern, and H.-Y. Lin, Opt. Express 19, 415 (2011).

${ }^{14}$ N. I. Landy, C. M. Bingham, T. Tyler, N. Jokerst, D. R. Smith, and W. J. Padilla, Phys. Rev. B 79, 125104 (2009).

${ }^{15}$ Y. Qiu Xu, P. Heng Zhou, H. Bin Zhang, L. Chen, and L. Jiang Deng, J. Appl. Phys. 110, 044102 (2011).

${ }^{16}$ X. Huang, H. Yang, S. Yu, J. Wang, M. Li, and Q. Ye, J. Appl. Phys. 113, 213516 (2013).

${ }^{17}$ B. Wang, T. Koschny, and C. M. Soukoulis, Phys. Rev. B 80, 033108 (2009).

${ }^{18}$ J. Grant, Y. Ma, S. Saha, A. Khalid, and D. R. S. Cumming, Opt. Lett. 36, 3476 (2011).

${ }^{19}$ S. Chen, H. Cheng, H. Yang, J. Li, X. Duan, C. Gu, and J. Tian, Appl. Phys. Lett. 99, 253104 (2011).

${ }^{20}$ H. Luo, X. Hu, Y. Qiu, and P. Zhou, Solid State Commun. 188, 5 (2014).

${ }^{21}$ D. Viet, N. Hien, P. Tuong, N. Minh, P. Trang, L. Le, Y. Lee, and V. Lam, Opt. Commun. 322, 209 (2014).

${ }^{22} \mathrm{~S}$. Li, J. Gao, X. Cao, W. Li, Z. Zhang, and D. Zhang, J. Appl. Phys. 116, 043710 (2014).
${ }^{23}$ G.-H. Yang, X.-X. Liu, Y.-L. Lv, J.-H. Fu, Q. Wu, and X. Gu, J. Appl. Phys. 115, 17E523 (2014).

${ }^{24}$ B.-Y. Wang, S.-B. Liu, B.-R. Bian, Z.-W. Mao, X.-C. Liu, B. Ma, and L. Chen, J. Appl. Phys. 116, 094504 (2014).

${ }^{25}$ C. Sabah, F. Dincer, M. Karaaslan, E. Unal, O. Akgol, and E. Demirel, Opt. Commun. 322, 137 (2014).

${ }^{26}$ J. Lv, R.-y. Yuan, X. Song, and H. Yan, Journal of Applied Physics 117, 013101 (2015).

${ }^{27}$ C. Shi, X. Zang, Y. Wang, L. Chen, B. Cai, and Y. Zhu, Appl. Phys. Lett. 105, 031104 (2014).

${ }^{28}$ J. Zhu, Z. Ma, W. Sun, F. Ding, Q. He, L. Zhou, and Y. Ma, Appl. Phys. Lett. 105, 021102 (2014).

${ }^{29}$ F. Ding, L. Mo, J. Zhu, and S. He, Appl. Phys. Lett. 106, 061108 (2015).

${ }^{30}$ P. Bouchon, C. Koechlin, F. Pardo, R. Haïdar, and J.-L. Pelouard, Opt. Lett. 37, 1038 (2012).

${ }^{31}$ N. Liu, M. Mesch, T. Weiss, M. Hentschel, and H. Giessen, Nano Lett. 10, 2342 (2010).

${ }^{32}$ C. Hua, S. Chen, Y. Haifang, L. Junjie, X. An, C. Gu, and J. Tian, J. Opt. 14, 085102 (2012).

${ }^{33}$ Y. Song, C. Wang, Y. Lou, B. Cao, and X. Li, Opt. Commun. 305, 212 (2013).

${ }^{34}$ Y. E. Kesim, E. Battal, and A. K. Okyay, AIP Adv. 4, 077106 (2014).

${ }^{35}$ F. Cheng, X. Yang, and J. Gao, Opt. Lett. 39, 3185 (2014).

${ }^{36}$ J. Hendrickson, J. Guo, B. Zhang, W. Buchwald, and R. Soref, Opt. Lett. 37, 371 (2012).

${ }^{37}$ S. Ogawa, D. Fujisawa, H. Hata, M. Uetsuki, K. Misaki, and M. Kimata, Appl. Phys. Lett. 106, 041105 (2015).

${ }^{38}$ C. Argyropoulos, K. Q. Le, N. Mattiucci, G. D'Aguanno, and A. Alù, Phys. Rev. B 87, 205112 (2013).

${ }^{39}$ Y. Ma, Q. Chen, J. Grant, S. C. Saha, A. Khalid, and D. R. S. Cumming, Opt. Lett. 36, 945 (2011).

${ }^{40} \mathrm{P}$. Markoš and C. M. Soukoulis, Wave Propagation (Princeton University Press, 2008), p. 376.

${ }^{41}$ D. R. Smith and J. B. Pendry, J. Opt. Soc. Am. B 23, 391 (2006).

${ }^{42}$ C. M. Bingham, H. Tao, X. Liu, R. D. Averitt, X. Zhang, and W. J. Padilla, Opt. Express 16, 18565 (2008).

${ }^{43}$ Q.-Y. Wen, H.-W. Zhang, Y.-S. Xie, Q.-H. Yang, and Y.-L. Liu, Appl. Phys. Lett. 95, 241111 (2009).

${ }^{44}$ X. Liu, T. Tyler, T. Starr, A. F. Starr, N. M. Jokerst, and W. J. Padilla, Phys. Rev. Lett. 107, 045901 (2011).

${ }^{45}$ Y. Cheng, Y. Nie, and R. Gong, Opt. Laser Technol. 48, 415 (2013).

${ }^{46} \mathrm{See}$ http://www.comsol.com for COMSOL Multiphysics.

${ }^{47}$ M. A. Ordal, R. J. Bell, R. W. Alexander, L. L. Long, and M. R. Querry, Appl. Opt. 26, 744 (1987).

${ }^{48}$ See http://www.surfaceoptics.com for Surface Optics Corporation.

${ }^{49}$ V. K. Kamineni, P. Singh, L. Kong, J. Hudnall, J. Qureshi, C. Taylor, A. Rudack, S. Arkalgud, and A. C. Diebold, in 5th International Conference on Spectroscopic Ellipsometry (ICSE-V) [Thin Solid Films 519, 2924 (2011)].

${ }^{50}$ W.-C. Chen, M. Koirala, X. Liu, T. Tyler, K. G. West, C. M. Bingham, T. Starr, A. F. Starr, N. M. Jokerst, and W. J. Padilla, e-print arXiv:1212.2868 [physics.optics]. 